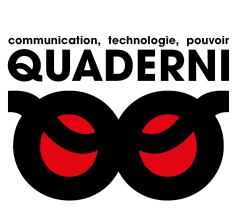

\title{
Quaderni
}

Communication, technologies, pouvoir

98 | Hiver 2018-2019

Humanités numériques : vers l'institutionnalisation

\section{Usages numériques et nouvelles relations muséales}

Digital uses and new relationships to museum data

\section{Geneviève Vidal}

\section{(2) OpenEdition}

\section{Journals}

Édition électronique

URL : https://journals.openedition.org/quaderni/1443

DOI : 10.4000/quaderni.1443

ISSN : 2105-2956

\section{Éditeur}

Les éditions de la Maison des sciences de l'Homme

Édition imprimée

Date de publication : 5 février 2019

Pagination : 61-72

\section{Référence électronique}

Geneviève Vidal, « Usages numériques et nouvelles relations muséales », Quaderni [En ligne], 98 | Hiver 2018-2019, mis en ligne le 05 février 2021, consulté le 08 janvier 2022. URL : http://

journals.openedition.org/quaderni/1443; DOI : https://doi.org/10.4000/quaderni.1443 


\section{$D$ ossier}

\section{Usages numériques et nouvelles relations muséales}

À l'heure des innovations numériques, du web 3.0 avec l'émergence de l'internet des objets, du web des données, du web sémantique, provoquant des traitements de données massives, nous proposons de ne pas surdéterminer la technologie, mais de considérer les interactions entre acteurs humains et non humains producteurs de données et de contenus.

\section{Geneviève Vidal}

Maître de Conférences (HDR) Université Paris 13

Avant d'engager l'analyse de ces interactions et leurs implications et conséquences, nous distinguons les données des contenus, à un premier niveau : les machines traitent des données, les humains produisent des contenus. À un second niveau, en créant des contenus, les humains produisent des données, traitées par les machines produisant à leur tour des données.

Dans ce contexte de la culture de la donnée ${ }^{1}$ et ces conditions, il importe de tenir compte du rôle des médiations, numériques et humaines, puisque les interactions de pratiques, abordées en première partie, se confirment grâce à des médiations. Pour mener à bien cette analyse des interactions entre des pratiques numériques et culturelles de la part d'acteurs de données et de contenus, nous faisons l'hypothèse que la médiation offre le cadre d'usages numériques ouvrant sur de nouvelles relations aux données, abordées en seconde partie, en stock (bases de données) et en flux (réseaux), dans des environnements pervasifs. Le cas des données émanant du ou en lien avec le secteur muséal est heuristique dans la mesure où ce dernier constitue un observatoire des usages numériques contemporains. 


\section{Usages en interactions}

Nous considérerons les interactions de pratiques numériques et culturelles dans le cadre de la rencontre entre un réseau centralisé -les muséeset un réseau décentralisé - l'Internet - dont la figure stimule des innovations culturelles et organisationnelles. En analysant les usages numériques professionnels et ceux des publics des médiations numériques muséales, nous relevons de récents engagements, parfois audacieux, et des mouvements endogène et exogène de ressources et contenus de la part des musées et des publics.

Quatre thèmes d'analyse peuvent être retenus pour cerner les enjeux de ces pratiques et usages en interactions : 1) les frontières des usages des médiations numériques muséales, 2) leurs significations, les sociabilités et émotions, 3) les stratégies des institutions pour penser des flux de données, ces trois thèmes ouvrant sur 4) les relations publics et musées, et les relations aux contenus patrimoniaux et scientifiques.

Les études qualitatives d'usages de médiations numériques muséales permettent de considérer le secteur muséal comme un observatoire des innovations numériques et de la formation d'un big data culturel. En effet, les technologies numériques, aux formes diversifiées - des interfaces fonctionnelles aux interfaces mobiles et tactiles - donnent lieu à une circulation massive de données de la part des institutions et de leurs publics. Ces technologies sont mises en œuvre pour instaurer une mise en relation avec les contenus scientifiques, historiques, artistiques et patrimoniaux, et entre les institutions et leurs publics. Les musées contribuent ainsi à la confirmation du web participatif et du web des données.

Les récents usages participatifs sur les sites web institutionnels et les réseaux sociaux numériques correspondent à une approche qui consiste à recueillir les données des usagers, quand les médiations sollicitent une expression avec des appels à dessins, photographies, tags, commentaires, voire stimulent des dynamiques relevant des sciences participatives ${ }^{2}$. Mais les musées doivent aussi considérer sur l'Internet des témoignages et expériences, en relation avec leurs activités, ressources et collections, avec des textes, vidéos et images d'amateurs qui n'attendent pas leurs invitations et appels à participation. Dans les musées, nous avons relevé les mouvements de textes et d'images qui entrent et sortent des dispositifs personnels des visiteurs ${ }^{3}$, contribuant à un rayonnement des contenus muséaux.

La démarche d'étude doit penser des usages mobiles et fluctuants, dont les objectifs peuvent évoluer au fil du temps, comme c'est le cas des blogs recueillant peu de commentaires et qui ont tendance récemment à donner la parole à des amateurs éclairés devenant partenaires des institutions pour nourrir l'Internet muséal. Étudier les usages numériques ne se réduit pas à l'évaluation de la satisfaction, des utilisations ergo-cognitives. Tout en en tenant compte, la démarche permet aussi l'analyse de leurs significations, notamment grâce à l'étude des interactions de pratiques culturelles, communicationnelles et numériques, marquant l'appropriation des médiations muséales. Il faut 
cerner les modes d'intervention des publics, les sociabilités qui fournissent des données sur les comportements, grâce aux techniques de traçabilité avec des algorithmes, ce qui est avantageux pour le chercheur qui vise la constitution de corpus conséquents pour mener à bien sa recherche. Aussi est-il indispensable d'adopter une éthique à l'égard du traitement et de l'exploitation possible de ces données ${ }^{4}$. Les chercheurs travaillant sur et avec l'Internet se dotent petit à petit de chartes pour réguler cette capture de contenus et de comportements en ligne. Les professionnels de musée de plus en plus attentifs aux usages sur l'Internet sont également amenés à s'engager dans cette voie vigilante et rigoureuse afin de poursuivre l'élaboration des médiations numériques connectées.

Les visiteurs porteurs de smartphones et de tablettes connectés à l'Internet peuvent créer une mise en réseau de contenus et de données, provenant du musée lui-même, avec des dispositifs sophistiqués comme les tables interactives multitouches et les dispositifs multi-écrans ou avec des applications mettant en œuvre la médiation en réalité augmentée. Les publics font circuler leurs témoignages relatifs à leurs usages sur les réseaux sociaux numériques et auprès de leurs contacts par messagerie électronique et messages courts.

Nous constatons que ce mouvement faisant entrer et sortir des données et des contenus, mettant en relation d'une nouvelle manière les publics et les musées, dépasse la notion d'interactivité en termes strictement ergocognitifs. L'enjeu, à l'heure de l'accès à des données massives patrimoniales et scientifiques, rend compte d'une multiplicité et d'une plasticité des compétences et des significations d'usages, pour les publics de la culture, et des réseaux de communication.

Prompts à mobiliser leur équipement personnel pour opérer ces mouvements de contenus et données, les visiteurs apprécient les expositions qui les associent, les musées qui les placent partenaires de la médiation. Aussi engagent-ils un regard critique sur les technologies quand des limites d'ordre ergonomique se présentent. Les expressions critiques, voire des abandons, sont à relever quand des configurations innovantes d'usages sont considérées complexes, comme un double-écran ou un environnement pervasif prenant appui sur le wifi, la rfid pour retrouver les contenus en liaison avec un jeu dans une exposition. Néanmoins ces innovations suscitent des solidarités relevant d'inventions d'usages, qui font sens pour les visiteurs.

Grâce à leurs usages réflexifs, les visiteurs déplorent aussi des contenus insuffisamment approfondis, des enchaînements entre la médiation numérique et la collection exposée ou des redondances entre les différentes médiations, au fil de la visite appareillée (IPad, wifi, carte RFID par exemple) ${ }^{5}$. Ils se désintéressent en outre des médiations en deçà $\mathrm{du}$ potentiel technique, les bornes notamment considérées obsolètes, en se référant à leurs pratiques communicationnelles pour convoquer des contenus.

Malgré ces critiques, des visiteurs peuvent accorder de longues minutes à une consultation 
multimédia quand un objectif relié à une histoire personnelle est fixé. Autrement dit, il nous faut entendre une ambivalence avec une exigence d'autonomie avec les médiations, ou une mise en débat, et parallèlement une volonté de ne pas être trop actifs, d'être guidés de façon intuitive, autrement dit sans mode d'emploi, en s'appuyant sur l'expérience numérique.

Entre frustration et curiosité, les visiteurs apprécient de pouvoir choisir les médiations pour vivre leur expérience de visite comme ils l'entendent, pouvant aller jusqu'à se préoccuper de questions auxquelles le musée ne répond pas. Aussi, souhaitent-ils des extensions du discours du musée, selon des modalités relevant de la réalité augmentée, dans un non dit, et à partir des interactions de pratiques dont ils font preuve, en menant une recherche avec un moteur de recherche sur l'Internet ou sur l'encyclopédie collaborative Wikipedia.

Ces médiations ainsi que les limites critiquées déclenchent l'envie d'aller plus loin après la visite de musée, dans le cadre de pratiques de recherches documentaires et pratiques culturelles étendues. Les médiations jouent un rôle qui dépasse la diffusion d'un message ou d'informations au sein de l'institution ou à l'extérieur avec une application mobile. Les médiations suscitent des émotions relatives aux connaissances préalables et nouvelles, ainsi qu'aux histoires personnelles.

C'est particulièrement le cas quand le musée propose des jeux via des dispositifs mobiles (téléphones personnels ou tablettes) qui vont rythmer les visites. Ce type de médiations stimule des émotions au cœur des relations entre les visiteurs et lors des déplacements dans l'espace de l'exposition ponctuée d'autres émotions avec les collections et les discours scientifiques. Les invitations à participer sont alors accueillies par les visiteurs à la recherche de réponses ou contenus dans l'exposition par le truchement de la médiation numérique. Les publics vont par ailleurs apprécier les applications permettant un prolongement de l'expérience de visite à distance (sur l'Internet, avec une application).

Durant la visite, une disposition à l'innovation est ainsi confirmée avec les équipements personnels des visiteurs (appareils photos numériques, smartphones, tablettes), qui diffusent des contenus du musée à l'extérieur, pour les montrer à leurs proches ou amis. Se mêlent alors des pratiques culturelles et communicationnelles, dessinant de nouvelles frontières à l'exposition muséale. Ce double mouvement exogène et endogène rejoint une masse de données et de contenus participant du big data culturel, qui ne fait que croître avec l'Internet des objets et le web sémantique, parallèlement à la poursuite du web participatif.

Les données circulent donc dans l'enceinte et hors du musée, grâce aux professionnels des musées et leurs publics partenaires de ces flux de données, au cœur desquels, les institutions établissent une proximité avec les publics de la culture. Mais les internautes, en quête d'une autonomisation de l'accès à la culture, souhaitent s'exprimer en tant qu'amateurs de thèmes patrimoniaux ou scientifiques. Les institutions cherchent alors à entrer en relation avec les amateurs en ligne ${ }^{6}$ en invitant à participer à leurs 
activités en ligne, comme c'est le cas de l'appel $\mathrm{du}$ Museum National d'Histoire Naturelle "Viginature $»^{7}$ qui consiste à recevoir des photographies d'amateurs d'insectes, de jardins, pour contribuer à une dynamique de recherche scientifique dans le cadre d'un crowdsourcing maîtrisé par l'institution. Mais, les amateurs, n'attendant pas les invitations d'institutions patrimoniales pour s'emparer de questions similaires à celles portées par les initiatives scientifiques, mettent en place des réseaux collaboratifs, en l'occurrence celui d'amateurs de botanique $«$ TelaBotanica $»^{8}$.

Des « campagnes de collectes » via Internet (prêts d'objets, dons, témoignages filmés), comme celles du Musée d'histoire de Nantes avec « Net collection »" sont également lancées. Un autre exemple concerne des portails relevant du web collaboratif avec par exemple le projet 《histoire2guerres ${{ }^{10}}^{10}$ créé et alimenté par le Musée d'histoire de Nantes en relation avec seize institutions et associations du département de Loire-Atlantique. Ou encore le cas, interrompu désormais, du Art Museum Social Tagging Project ${ }^{11}$ piloté par des professionnels de musées américains comme le Guggenheim Museum, le Los Angeles County Museum of Art, le Metropolitan Museum of Art, en faveur de la dynamique de la folksonomie.

Le territoire muséal sur l'Internet $^{12}$ donne également à voir des expérimentations, d'où découle une profusion de données rejoignant le big data culturel, telle que la co-conception d'expositions ${ }^{13}$, allant parfois jusqu'à la confiance radicale (radical trust) ${ }^{14}$. Ces innovations participatives-contributives portées par les amateurs, profanes ou experts, et les musées ne relèvent pas nécessairement de stratégies marketing, pour autant ce qui a été nommé jusqu'ici le web 2.0 consiste à offrir des outils de mise en contact servant un marketing culturel.

Au delà de l'approche marketing, l'objectif du web participatif par le biais des sites institutionnels, notamment blogs et plateformes patrimoniales institutionnelles ou les « comptes » de musées sur des réseaux sociaux numériques exogènes comme twitter facebook, youtube, flickr par exemple, reste la diffusion de la culture scientifique, historique, artistique, patrimoniale auprès d'un public à élargir, ainsi que la valorisation des ressources. Dans ce cadre diffusionniste, la mise à disposition de services dits encore 2.0 par les institutions muséales permet aux publics d'intervenir sur le fonds (œuvres et contenus scientifiques) et la forme (avec la personnalisation des espaces personnels), d'échanger, de décrire (en indexant avec des tags, en annotant avec des dispositifs d'annotations) et parfois de contribuer. Ces usages recoupent les fonctions d'informationcommunication, de médiation et la fonction sociale du musée.

Ainsi, la multimédiatisation des données de la sphère muséale suscite de nouveaux accès mais aussi des mouvements et interactions de données relatives aux activités des institutions muséales, à leurs collections et aux connaissances dans le champ des arts, de l'histoire, du patrimoine et des sciences. Cette configuration donnant lieu à un big data muséal ouvre sur une réflexion concernant $l^{\prime}$ Internet comme bien 
public commun ${ }^{15}$. Nous pouvons également relever, dans un contexte d'échange numérique généralisé, l'émergence d'innovations organisationnelles au sein des institutions fondées sur la mixabilité des compétences. Nonobstant, les technologies participatives, contributives, collaboratives semblent ne pas modifier la conception de l'autorité du musée comme détenteur et diffuseur de connaissances.

L'internet muséal donne une nouvelle visibilité aux musées, qui peuvent se concevoir dans une chaîne d'échanges massifs avec différents publics auteurs de contenus. Ces flux de données des musées et des internautes (publics de musées ou non) étendent leurs relations dans le cadre d'une culture numérique qui s'élabore et forme une base d'interactions de pratiques traversées par le numérique. Nous allons maintenant aborder les relations entre musées et publics, qui témoignent de la formation d'un web des données.

\section{Relations entre publics et musées, et web des données}

De nouvelles relations entre publics et musées ouvrent sur une nouvelle façon de penser la diffusion muséale dans le contexte du développement du web des données. Dès lors, des stratégies numériques mais aussi économiques donnent lieu à des partenariats ${ }^{16}$ entre musées et d'autres institutions, pour penser les flux de données structurées. Les institutions engagent en effet une politique de sémantisation de leurs données, l'élaboration de métadonnées et ontologies ${ }^{17}$ pour offrir un accès aux ressources selon une interopérabilité et en rupture avec des consultations linéaires et arborescentes, telles qu'elles ont été jusqu'à présent pensées selon des logiques relatives aux bases de données servant des usages savants.

Parallèlement à cette élaboration sophistiquée, les productions de contenus de la part des amateurs des sciences et du patrimoine se poursuivent sur l'Internet. Aussi, pour gérer l'abondance, les musées régulent la production de contenus de la part des publics, tout en faisant face à l'économie de leurs médiations numériques. Tant pour les institutions que pour les prestataires des applications et dispositifs, il s'agit de réduire les coûts. Aussi, la reproductibilité, le partage d'expériences, les partenariats inter-institutions, en termes de ressources, de droits, en termes financiers et d'usages, visent une économie des politiques numériques des musées.

La mise en œuvre du web des données, encore en cours, ne doit cependant pas négliger les pratiques numériques avec l'ambivalence d'usages et la posture critique des publics de musées qui participent des relations entre les musées et les publics. Ces pratiques en interactions ouvrent sur une conception d'action sur les contenus jusqu'à l'apport de contenus de la part des publics, ne signifiant pourtant pas la fin des rapports inégalitaires à la culture, ni des rapports de domination des prescriptions. Les relations publics et musées participent des expérimentations d'innovations, alternatives au modèle de l'exposition. Les médiations numériques reposent sur le participatifcontributif et la mobilité, grâce aux dispositifs personnels des visiteurs et à ceux de l'institution. 
Récemment, une profusion d'applications mobiles émerge, et un éventuel transmedia ${ }^{18}$ muséal et des dispositifs de réalité virtuelle sont précédés par des reconstitutions $3 \mathrm{D}$ plébiscitées, mettant en valeur la recherche patrimoniale et scientifique.

Pour l'heure, se développent surtout les applications mobiles dans lesquelles les contenus patrimoniaux et scientifiques correspondent aux standards de contenus courts et ludiques avec des scénarios, des jeux et des ergonomies similaires à ceux d'autres applications sans lien avec les musées. Ces applications doivent de plus, selon notre étude d'usages d'applications mobiles ${ }^{19}$, faire évoluer la voix, les ambiances sonores pour instaurer une narration dite immersive. Il est en outre important de penser le champ lexical en lien avec la richesse du fonds de l'institution muséale. Pour la conception-réalisation des applications mobiles, les institutions muséales et leurs prestataires cherchent à capter l'attention des publics de la culture et de nouveaux publics, notamment ceux ne fréquentant pas les lieux patrimoniaux, comme des jeunes publics à la recherche de scénarios, de jeux et d'ergonomies similaires à ceux d'autres applications non reliées aux contenus patrimoniaux. Ces publics se réfèrent à des représentations de technologies numériques permettant la liberté de choix de contenus, l'intuitif, avec une rapidité de traitements des commandes, la prise en compte $\mathrm{du}$ contexte d'usages et des points de vue (tant dans le site patrimonial qu'à l'extérieur), avec la géolocalisation.

Les professionnels du secteur patrimonial sont par ailleurs amenés à céder au modèle économique des médiations innovantes fondées sur l'obsolescence programmée des technologies. Les résultats de l'étude des usages d'applications in situ et à distance nous permettent d'identifier trois ordres d'enjeux des transformations des rapports au patrimoine et aux connaissances :

- la façon d'envisager, d'organiser les contenus patrimoniaux par le prisme du numérique ;

- les pratiques professionnelles et les pratiques des publics du patrimoine culturel ;

- un processus d'industrialisation-marchandisation des contenus patrimoniaux numériques, provoquant des résistances, l'importation des logiques économiques et des modes de travail, plus ou moins éloignés des missions patrimoniales, issues du marché du numérique et des innovations.

L'ouverture des applications pour permettre des usages multi-supports, des relations de partage et d'échanges avec les publics et entre les usagers pour considérer les sociabilités numériques et l'autonomisation d'usages numériques, voire entre publics et chercheurs, est envisageable. Mais les professionnels ciblent prioritairement le ludo-éducatif avec des scénarios reposant sur le storytelling, afin de conduire les publics à travers un parcours de connaissances dans le site patrimonial ou via les médiations outdoor. De fait, il est plutôt envisagé de porter à la connaissance, avec des médiations prescriptives et de mener une politique de diffusion patrimoniale tout en valorisant les usages numériques, voire la créativité des publics.

L'équation n'est pas simple et pour les acteurs du marché du numérique, la solution est 
l'industrialisation, en proposant aux institutions de composer leurs propres applications à partir d'une fourniture de bases de données et de services standardisés.

Pour les institutions muséales, les orientations stratégiques, tant pour la recherche que pour leur politique de publics, convergent vers le web sémantique ${ }^{20}$. Les technologies de sémantisation des données, des objets connectés et de l'internet des objets, dépassant de fait les applications "one shot » trop coûteuses, permettraient aux musées de maîtriser la situation de communication, de confirmer l'autorité institutionnelle, de rationaliser la profusion, de rapprocher et mettre à distance les expériences hétérogènes des publics, usagers et consommateurs d'offres numériques, couplées à des algorithmes qui tracent les activités, dont celles sur l'Internet muséal.

Le big data culturel est cependant une chance pour la démocratisation de l'accès à la culture, qui dans le même temps sert la recherche. Ces données massives patrimoniales et scientifiques s'inscrivent dans un web des données et dans le processus de sémantisation des données, pour ainsi rejoindre la dynamique du web 3.0.

\section{Perspectives}

Les études d'usages des médiations numériques muséales éclairent l'expérience numérique en musée, avec une disposition à l'innovation de la part des publics dont les usages sont ambivalents ; entre mise en débat et critique des médiations numériques et demande de prescriptions fondées sur la légitimité de l'institution muséale. Les pratiques numériques et culturelles en interactions des publics donnent lieu à un réseau de données hétérogènes. Ces dernières proviennent de l'intérieur et de l'extérieur des musées, par le truchement de dispositifs fournis par les institutions et des équipements personnels des visiteurs-usagers. Les musées veulent prioritairement poursuivre leur mission de diffusion sans négliger leur politique de communication numérique pour la valorisation de leurs ressources et activités. Ils mettent en place de nouveaux partenariats pour maîtriser leurs productions multimédias en visant une économie de leurs politiques numériques et de médiation.

Dès lors, les données des publics et celles des institutions composent un web des données, à savoir un maillage de données structurées, afin de faciliter leur circulation, les échanges et traitements. En effet, les publics souhaitent pouvoir interagir avec les objets et données, approfondir certaines connaissances, y compris en dehors du musée, mais aussi participer à la circulation des données, enrichir les contenus et communiquer sur leurs expériences de visite et culturelles.

En ligne, les musées à l'écoute de leurs publics engagent, après une phase d'appropriation professionnelle des technologies numériques, une rationalisation des interventions des internautes et leur régulation grâce à une sophistication technique et éditoriale des médiations numériques. Ils consolident leurs savoir-faire professionnels tels que l'indexation et la structuration des données. Le web sémantique, prôné capable de prendre en charge, 
grâce aux calculs, navigations et consultations, devrait par ailleurs permettre au secteur muséal de conserver ses prérogatives, tout en permettant des liens sémantiques.

Parallèlement au déploiement des nouvelles médiations numériques, les activités de recherche s'appuient sur les bases de données de collections et sur la structuration des données massives numériques patrimoniales et scientifiques. Les partenariats professionnels facilitent une mutualisation des compétences, des moyens et des fonds, dans un contexte de restriction budgétaire pour produire des contenus, des hyperliens sémantiques entre les données, circulant dans les réseaux. La formation d'un web 3.0 est ainsi en marche, incluant désormais d'autres données culturelles d'institutions comme les opéras, théâtres et monuments, qui ouvrent sur l'élaboration d'innovations culturelles.

\section{$R \cdot \dot{E} \cdot F \cdot E \cdot R \cdot E \cdot N \cdot C \cdot E \cdot S$}

DELMAS-MARTY Mireille, 2012, "Foreword ", in Massit-Follea Françoise, Meadel Cécile, Monnoyer-Smith Laurence, Normative Experience in Internet Politics, Paris, Presses des Mines, coll. Sciences sociales, p. 11-18.

MANOVITCH Lev, 2005, " Remixability and Modularity », en ligne : http://manovich. net/index.php/projects/remixability-andmodularity.

MASSIT-FOLLÉA Françoise, 2011, « Usages et Gouvernance de l'Internet : pour une convergence socio-politique ", in Vidal Geneviève (dir.), La sociologie des usages : continuités et transformations. London: Hermes Science Publishing, p. 153-178.

PROULX Serge et LATZKO-TOTH Guillaume, 2013, « Enjeux éthiques de la recherche sur le Web ", in Barats Christine (dir.), Manuel d'analyse $d u$ web, Paris, Armand Colin, collection U Sciences humaines et sociales, chapitre 2, p. 32-52.

SPADACCINI Jim et SEBASTIAN Chan, 2007, « Radical Trust: The State of the Museum Blogosphere », in Trant Jennifer and Bearman David (eds), " Museums and the Web 2007 », Proceedings, Toronto, Archives \& Museum Informatics, published March 31, Toronto, en ligne : http://www.museumsandtheweb.com/ mw2007/papers/spadaccini/spadaccini.html.

VIDAL Geneviève, 2009, "Le web 3.0, pour en finir avec le web 2.0 ? », Documentation et bibliothèques, vol. $55 \mathrm{n}^{\circ} 4$, octobre-décembre 2009, p. 201-207. 
$\mathrm{N} \cdot \mathrm{O} \cdot \mathrm{T} \cdot \mathrm{E} \cdot \mathrm{S}$

1. Voir parmi la littérature et les documents professionnels sur la culture de la donnée ou dite aussi culture des données : Collet-Thireau, K. et Thomas, J., 2015, « Big Data et Open Data : quel impact pour les professionnels de l'information ?», I2D - Information, données \& documents, volume 53, (4), p. 9-10, en ligne : https://www.cairn.info/revuei2d-information-donnees-et-documents-2015-4page-9.htm.

2. Voir : http://www.sciences-participatives.com.

3. L'exemple d'un visiteur (homme, 68 ans, bordelais) du Musée d'histoire de Nantes montre bien le mouvement d'images provoqué par les publics : «Ben je vais la montrer à ma famille, on est Bordelais, nous aussi on en a fait de belles, faut pas oublier et assumer, et comment on assume, en en parlant. J'ai appris pendant ce séjour à Nantes que Bordeaux est la deuxième capitale de la traite des noirs ».

4. Les opérations de traçabilité, calculs et modélisation de données par recoupements de bases gigantesques, inscrites dans le dit big data, sont de plus en plus courantes dans le cadre de l'économie numérique, notamment l'économie du gratuit ou des téléchargements d'applications à bas prix.

5. Exemple d'étude d'usages numériques en musée : Vidal Geneviève, avec la collaboration de Cecilia Jauniau, Ilaria Valoti, Anne Gagnebien, 2011, «Usages de dispositifs de médiation multimédia : tablette tactile et puces rfid; table multitouch », Exposition Le Musée des Confluences dévoile ses réserves, présentée au Musée Gallo-Romain de Fourvière -Lyon, 16 décembre 2010-8 mai 2011, LabSic - Université Paris 13, Musée des Confluences. 6. Voir la thèse de Lisa Chupin « Enjeux communicationnels de la conception de dispositifs de médiation documentaire augmentée pour les herbiers numérisés », soutenue le $1^{\text {er }}$ décembre 2017, CNAM, Laboratoire DICEN, École doctorale Abbé Grégoire, notamment l'analyse du site de sciences participatives «Les Herbonautes » avec le Muséum National d'Histoire Naturelle.

7. http://vigienature.mnhn.fr/

8. http://www.tela-botanica.org/

9. http://www.chateau-nantes.fr/fr/collections/net collections/

10. http://www.chateau-nantes.fr/fr/expositions/ autour_de_1_exposition_en_guerres/portail_ histoire_2_guerres/

11. Site dédié désormais inaccessible : http://www. steve.museum/

12. Vidal Geneviève, Hadid Sabrina, 2011, « Le territoire culturel sur les réseaux numériques. Le cas des musées », communication lors des Journées d'étude « Du web social au développement des territoires », des 6-7 octobre 2011, du groupe SFSIC Ticis, École des Mines de Nîmes-Alès.

13. Le Brooklyn Museum a, dès les années 2000, expérimenté la co-conception d'expositions avec ses publics : http://www.brooklynmuseum.org/ community/. L'événement Museomix quant à lui expérimente la co-conception, avec des acteurs professionnels diversifiés, de médiations et d'applications numériques http://www.museomix. com/.

14. Spadaccini, J. et C. Sebastian, (2007). "Radical Trust: The State of the Museum Blogosphere". In J. Trant and D. Bearman (eds). "Museums and the Web 2007”: Proceedings. Toronto: Archives \& Museum Informatics, published March 31, 2007, http:// www.archimuse.com/mw2007/papers/spadaccini/ spadaccini.html

15. Delmas-Marty Mireille, 2012, «Foreword », in Massit-Follea Françoise, Meadel Cécile, Monnoyer- 
Smith Laurence, Normative Experience in Internet Politics, Paris, Presses des Mines, coll. Sciences sociales, p. 11-18. Voir aussi Le Crosnier Hervé, tic\&société, vol. $12, \mathrm{n}^{\circ} 1,1^{\mathrm{er}}$ semestre 2018, en ligne : http://journal.openedition.org/ticetsociete/2348.

16. Des accords sont établis comme celui signé entre le ministère de la Culture, Wikimedia et INRIA, Institut National de Recherche en Informatique et Automatique. La signature de ce partenariat le 19 novembre 2012 donne lieu à un certain mouvement favorable aux contenus validés par les institutions en lien avec l'encyclopédie collaborative Wikipedia, avec une vigilance à l'égard de la communication ouvrant sur une écriture collective : http://www.wikimedia. fr/signature-du-partenariat-semanticpedia-avecle-minist $\% \mathrm{C} 3 \% \mathrm{~A} 8 \mathrm{re}-\mathrm{de}-\mathrm{la}$-culture-et-inria. Voir également http://portail-joconde.over-blog.com/ article-jocondelab-premiers-pas-de-joconde-sur-leweb-semantique-119255498.html

17. Voir le modèle conceptuel de référence CIDOC CRM pour concevoir dès la phase de production une structuration des données interopérables avec ce modèle d'échange et de diffusion dans le champ patrimonial.

18. Le transmédia vise des usages sur différents médias collectifs ou personnels, fixes ou mobiles grâce à un scénario qui évolue selon les contributions de la part des usagers. C'est à partir des années 2005, parallèlement au web 2.0 que le transmedia, qui attirait dès la seconde moitié des années 1990 le monde de la publicité, est associé au storytelling pour créer une expérience dans un univers narratif (à distinguer du cross-media qui déploie une même narration sur différents médias), par exemple webseries, ARGalternative reality games, par une dite immersion participative.

19. Vidal Geneviève, 2015, « Résultats de l'étude d'usages d'applications mobiles numériques en relation avec le patrimoine culturel ", in Vidal Geneviève et Laroche Florent, " Modélisation et simulation du patrimoine : pour une évaluation critique des applications numériques », UDPN (usages des patrimoines numérisés).

20. http://www.w3.org/2001/sw/ 


\title{
$R \cdot E ́ \cdot S \cdot U \cdot M \cdot E ́$
}

La recherche sur les usages numériques offre de riches enseignements sur les pratiques des publics et les pratiques professionnelles dans le secteur muséal. La structuration des données massives patrimoniales et scientifiques ouvre sur des enjeux pour la recherche et pour l'appropriation sociale des technologies et des contenus culturels. Leur circulation, par les dispositifs de médiation dans l'enceinte des musées et sur l'Internet muséal, est assurée par les usages des publics de musées et des amateurs qui n'attendent pas les invitations institutionnelles à la participationcontribution. Ces mouvements in situ et sur l'Internet permettent de nouvelles relations au patrimoine et aux contenus scientifiques, dans un contexte d'innovations culturelles, sociales et scientifiques, et de la formation d'un big data culturel, servant également les pratiques savantes.

\begin{abstract}
Digital uses and new relationships to museum data Research on digital uses offers rich lessons on audience practices and professional practices in the museum sector. The structuring of massive heritage and scientific data opens up issues for research and for the social appropriation of technologies and cultural content. Their diffusion, inside and outside of museum, through the mediation, is ensured by the users who do not wait for institutional invitations to participate. Those movements inside and outside of museum allow new relationships to heritage and scientific content, in a context of cultural, social and scientific innovations, and the cultural big data, also serving scholarly practices.
\end{abstract}

